\title{
COMMENTARY
}

\section{Why the dimension matters in ecological models?}

\author{
¿Por qué importa la dimensión en modelos ecológicos?
}

\author{
RODRIGO RAMOS-JILIBERTO ${ }^{1,}{ }^{*}$, FRANCISCO HOECKER-ESCUTI ${ }^{2} \&$ JAIME MENA-LORCA $^{2}$ \\ ${ }^{1}$ Departamento de Ciencias Ecológicas, Facultad de Ciencias, Universidad de Chile, Casilla 653, Santiago, Chile \\ ${ }^{2}$ Mathematical Ecology Group, Instituto de Matemáticas, Universidad Católica de Valparaíso, Casilla 4059, Valparaíso, Chile \\ *Corresponding author: e-mail: roramos@uchile.cl
}

\begin{abstract}
In this work we discuss the ecological and mathematical significance of system's dimension in continuoustime population dynamics models. We show how the system's dimension reflects the ecological assumptions and affects both the spectrum of dynamic output and mathematical tractability of the models. We stress that the model dimension is not always the same as the number of state-variables, and we also present conditions under which the system's dimension is altered.
\end{abstract}

Key words: ODE, population dynamics, theoretical models, mathematical ecology, stability.

\section{RESUMEN}

En este trabajo discutimos la significación ecológica y matemática de la dimensión del sistema en modelos de dinámica poblacional en tiempo continuo. Mostramos cómo la dimensión del sistema refleja los supuestos ecológicos y afecta el espectro de resultados dinámicos así como la tratabilidad matemática de los modelos. Acentuamos que la dimensión de un modelo no es siempre equivalente al número de variables de estado, y presentamos condiciones bajo las cuales la dimensión del sistema es alterada.

Palabras clave: ecuaciones diferenciales ordinarias, dinámica poblacional, modelos teóricos, ecología matemática, estabilidad.

\section{INTRODUCTION}

Modeling ecological systems appeals for a number of decisions the modeler has to take in order to represent appropriately the phenomenon of interest. Many of these decisions relate to the degree of abstraction to be allowed in building a model, in the face of the questions posed and the system's nature. In this regard, the favored rule among theoretical ecologists seems always to be "keep the model as simple as possible" (e.g., Levins 1968, May 1974).

One fundamental decision for modeling a natural system is the number of state variables to include explicitly. Even though we were interested in the fate of a single variable (e.g., the time evolution of a population's size), the consideration or not of other interacting variables is a non-trivial choice. In this work we will discuss de meaning and the ecologicalmathematical significance of the model dimensionality in the frame of autonomous ordinary differential equation systems, and specifically how transitions from model systems of dimension-one to dimension-two, and from dimension-two to dimension-three and higher reflect implicit assumptions and affect both the spectrum of dynamic output and mathematical tractability of the models.

Although there exist several mathematical frameworks for modeling population dynamics, perhaps the most fundamental dichotomy stand for discrete versus continuous representations of natural processes. The choice between discrete and continuous models is far from be obvious and unambiguous, and we guess that much of the reasons for favoring one or another framework is a matter of technical convenience. Often it is accepted that continuous models should be used for modeling populations which exhibit continuous reproduction and overlapping generations, and discrete models for the opposite. Nevertheless, 
according to the time scale of observation living population will look as growing either continuously or discretely. From a philosophical point of view, some scientists assume that population dynamics is a continuous process and discretization can be made as an approximation over a finite time interval (Gurney \& Nisbet 1998). Others assume that real world is microscopically discrete and continuous representations are idealized pictures of that fact (Ginzburg \& Colyvan 2004). Whether life is fundamentally continuous or discrete, there exist two mathematical bodies of theory addressing each of these approaches and population ecology has developed through using both frameworks. Here, we benefit from the qualitative theory of differential equations for addressing our focus in the frame of continuous-time population models. Readers interested in knowing the advances on this field in the discrete framework could find valuable help in Royama (1992), Berryman (1999) and Turchin (2003).

Although most of the concepts presented here apply to any continuous-time biological model, our focus is on the field of theoretical population ecology and we will restrict our approach to deterministic unstructured continuous-time population models of the form

$$
\frac{d x}{d t}=f(x)
$$

where, $x \in \Omega \subseteq \Re^{n}$ and $f(x)$ represents the growth rate of a population of size $x$.

\section{DEFINING CONCEPTS}

Recent ecological literature make use of concepts such as dimension and order in a way that could lead to distraction, since they are defined within a variety of theoretical frameworks (see Berryman 1999, Turchin 2003, and Getz 2003) and the provided definitions not always agree with the usual dynamical-systems concepts. Therefore, we provide in this section the necessary definitions which will be used hereafter.

Berryman (1999) defines dimension in the frame of discrete autoregressive models, as the maximum time delay of the system. This definition is equivalent to that of process order made by Berryman (1999, based on Royama 1977) and Turchin (2003). On the other hand, Turchin (2003) defines the related term dynamical dimension (of an autoregressive model) as the number of state-variables included in the system.

In the above context, i.e., autoregressive models, the concept of dimension and process order are intimately related to both the proposed mechanism behind the observed dynamics: number of necessary variables (lagged densities) to adequately explain the dynamics, as well as the shape of the observed dynamics, e.g., first or second order oscillations.

Within the continuous-time framework, the definition of dynamical dimension provided by Turchin (2003) is incomplete, since it does not consider the actual degrees of freedom of the system. In other words, the number of state variables employed in a model can exceed the minimal number of variables needed to fully characterize the dynamics. For example, consider a classical epidemiological model (Gao et al. 1996):

$$
\begin{aligned}
& \frac{d X}{d t}=(b-d) X-\lambda X Y / N \\
& \frac{d W}{d t}=(\lambda X Y / N)-(d+\varepsilon) W \\
& \frac{d Y}{d t}=\varepsilon W-(\alpha+d) Y \\
& \frac{d N}{d t}=b X-d N-\alpha Y
\end{aligned}
$$

where variables represent the number of individuals who are susceptible $(X)$, exposed $(W)$, and infectious $(Y)$, in the population of size $N$. Since $N=X+W+Y$, the variables are not independent and the above model can be reduced to its equivalent

$$
\begin{aligned}
& \frac{d S}{d t}=b(1-S) S-(\lambda-\alpha) I S \\
& \frac{d I}{d t}=\varepsilon(1-S)-(\varepsilon+\alpha+b S-\alpha I) I \\
& \frac{d N}{d t}=(b S-d-\alpha I) N
\end{aligned}
$$

in which $S$ and $I$ are the fractions of susceptible and infectious, respectively. Therefore, the model of this example is three-dimensional 
even though it could be written in terms of four state-variables, in a similar fashion as when the number of parameters in a given model is reduced without changing the system behavior.

Mathematically speaking, the dimension of a system of differential equations corresponds to the dimension of the manifold to which the trajectories belong. In other words the system's dimension equals the minimal number of statevariables needed to determine the value, at any time, of all state-variables of the system. In ecological population models, the number of state-variables usually coincides with the dimension of the system since there is not direct interdependence among the variables. Nevertheless, in metapopulation models and many epidemiological ones (see for example Mena-Lorca and Hethcote 1992, Marquet \& Velasco-Hernández 1997, Mena-Lorca et al. 1999, Hethcote 2000), the dimension can be lower than the number of state-variables since the value of any state-variable can be obtained in terms of the others, as shown above.

On the other hand, we follow the usual definition of order of a differential equation system as " $m$ " if it contains an expression of the form,

$$
\frac{d^{m} x_{i}}{d t^{m}}
$$

being this the highest derivative that appears in the system. Note that, within some constraints, a differential equation of order $m$ can be reduced to a system of $m$ first-order differential equations. Therefore, the concepts of order and dimension are intimately related. Although population dynamics models of order higher than one are rather unusual, recently Ginzburg \& Colyvan (2004) have divulged a new framework to modeling single-species dynamics on the base of a second-order differential equation, which is analogous to a pair of first-order ODE. The model of Ginzburg \& Colyvan (2004) has the form

$$
\frac{d^{2} n}{d t^{2}}=F(n, r)
$$

and constitutes a two-dimensional system since contains two independent variables, say abundance $n$ and growth rate

$$
\frac{d n}{d t}=r
$$

\section{One-dimensional models}

A one-dimensional population model represents the rules for the growth rate of a single population. For example the system

$$
\frac{d x}{d t}=r x-b x^{2}-y c x
$$

represents the rate of change of the unique state-variable $x$ (population size) as a function of the variable itself, and parameters $r$ (intrinsic growth rate), $b$ (logistic self-limitation coefficient), $y$ (predators abundance), and $c$ (predation rate). Using signed digraphs ${ }^{1}$ - loop models - (Puccia \& Levins 1985), this system can be displayed as shown in Fig. 1A.

Note that equation (4) contains only one state-variable and thus the model has dimension one, even though there is a parameter $(y)$ representing the abundance of another population. We can easily rename the parameter $r$ as $r^{\prime}=r-y c$ and $K=r^{\prime} / b$, and model (4) changes to the well known form of the logistic equation.

It should be noted that one-dimensional models of any complexity contain only one state-variable, plus a certain number of parameters, provided that the system is autonomous (i.e. the right hand of the equation does not include explicitly the time as a variable). Main distinctions among onedimensional population models are the number of equilibrium points (i.e., when the growth rate is zero) that the systems can exhibit, and the functional relationships between the variable and parameters. In the case of polynomial models (i.e. when $f(x)$ in equation (1) has a polynomial structure), the number of

${ }^{1}$ Loop graphs show how a variable (represented by a vertex from which an arc starts) affects the rate of change of the variable to which is connected, provided that the system is close to an equilibrium. In other words, loop graphs are a picture of the Jacobian (community matrix), with each arc sketched from a variable $\mathrm{x}_{1}$ to another variable $\mathrm{x}_{2}$ representing

$$
\left.\frac{\partial}{\partial x_{1}}\left(\frac{d x_{2}}{d t}\right)\right|_{*}
$$

with $\mathrm{i}=1,2 \ldots n$, and $*$ is an equilibrium. An arc ending with an arrowhead indicates a positive effect, whereas an arc ending with a circle indicates a negative effect. Thus, Fig. 1A shows that the population size affects negatively its own rate of change near the equilibrium, for any positive value of the variable. 
equilibria is bounded by the degree of the polynomial. Equation (4) is a polynomial of degree-two, with the coefficient of the quadratic term being negative (self-limitation).

A one-dimensional model of degree-three is

$$
\frac{d x}{d t}=x r(1-b x)(x-m)
$$

which through modifying eq. (4) combines the negative feedback due to self-limitation and a positive feedback due to cooperation among population members (e.g., the Allee effect, see Dennis 1989, Stephens \& Sutherland 1999, Boukal \& Berec 2002, Berryman 2003).

It should be noted that non-polynomial models can be build by, for example, incorporating a nonlinear (such as those of the Holling type) predation function. Let us consider such modification through changing equation (4) to:
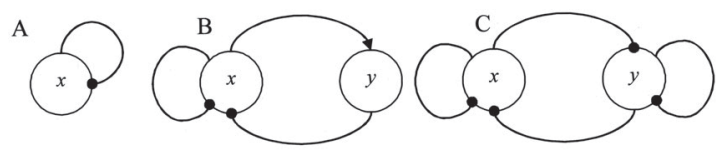

D

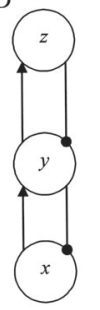

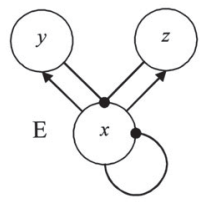

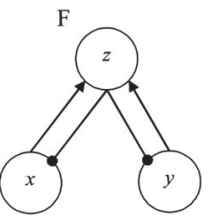

G

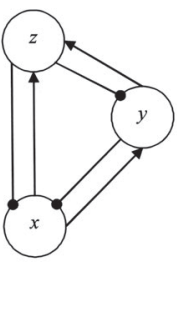

Fig. 1: Loop graphs of population systems. The circle represents the state variable; connectors ending in a small circle and an arrowhead indicate respectively a negative and a positive effect of one variable on the rate of change of the other. (A) Self-damped single population; (B) predator-prey interaction with prey self-limitation; (C) Lotka-Volterra competitive interaction; (D) trophic cascade; (E) exploitative competition; $(\mathrm{F})$ shared predator; $(\mathrm{G})$ intraguild predation.

Gráficos de loop de sistemas poblacionales. El círculo representa la variable de estado; los conectores que terminan en círculo pequeño y en punta de flecha indican respectivamente un efecto negativo y un efecto positivo de una variable sobre la tasa de cambio de la otra. (A) Población simple autolimitada; (B) interacción depredador-presa con autolimitación en las presas; (C) interacción competitiva de Lotka-Volterra; (D) cascada trófica; (E) competición explotativa; (F) depredador compartido; (G) depredación intragremio.

$$
\frac{d x}{d t}=r x-b x^{2}-\frac{y x^{2} c}{h+x^{2}}
$$

which make use of a Holling type-III predation function. The above equation can be studied through equation (7), which is a polynomial model of degree-four, since both are qualitatively equivalent.

$$
\frac{d x}{d t}=r x\left(h+x^{2}\right)-b x^{2}\left(h+x^{2}\right)-y x^{2} c
$$

On the other hand, models containing other kind of functions such as exponentials or power functions can be fairly approximated to a polynomial through a Taylor expansion near the equilibrium. Therefore, the local qualitative properties of non-polynomial models can be studied through their corresponding Taylor polynomial.

\section{Analysis of one-dimensional models}

In the Fig. 2 we plot the population growth rate as a function of $x$ according to the exponential, logistic, and Allee-effect models. These kind of plots provide valuable information about the fate of a trajectory, i.e. the time evolution of the state-variable. Their abbreviated forms, where the fluxes around equilibria are drawn along the $\mathrm{x}$-axis, are known as one-dimensional phase-portraits (Edelstein-Keshet 1988).

We can perform a direct stability analysis by just looking at the derivative of the growth rate

$$
\frac{\partial}{\partial x}\left(\frac{d x}{d t}\right)
$$

evaluated at equilibria. If negative, the equilibrium is locally stable; otherwise it is unstable (see Edelstein-Keshet 1988, Yodzis 1989, Chicone 1999, Kot 2001, Brauer \& Castillo-Chávez 2001).

Thus, one-dimensional systems can exhibit a limited range of dynamic behavior. First, it should be noted that the number of equilibrium points, if they exist, can be one or more. Second, an equilibrium point can be attractor or repellor but the transient trajectories are always monotonic. In other words, the fate of a population governed by a one-dimensional model is either increase or decrease, gently 
approaching the attracting equilibrium in the basin of attraction. Note that an attractor point could indeed be the zero, in which case the population approaches to extinction.

\section{Two-dimensional models}

Ecology deals with interactions, and for model many ecological processes it is necessary to include explicitly the dynamic interaction between two or more populations. Consequently, binary interactions such as predation, parasitism, competition, mutualism and others constitute foundational concepts in population ecology and hence they occupy an important fraction of most ecology textbooks.

Starting from the single species model (4), we can jump to a two-dimensional system through explicitly modeling the predator population. One alternative is

$$
\begin{aligned}
& \frac{d x}{d t}=r x-b x^{2}-y c x \\
& \frac{d y}{d t}=p y c x-m y
\end{aligned}
$$

where the growth rate of predators is governed by an input (sometimes interpreted as birth rate) and an output (death rate). In this case, the input is a fraction $p$ of food consumed and the output is an exponential decay. A more general interpretation is that the per capita growth rate of predators is a linear function of food consumed, with slope $p$ and intercept $-m$ (see Ginzburg 1998, Ramos-Jiliberto 2005). Of course, if the extraction rate of prey is modeled in other ways, the predator equation will change accordingly. Moreover, alternatives of converting prey consumed into predator own biomass other than a straight line also exist (see for example Getz 1991, 1993, Ramos-Jiliberto 2005). A graphical representation of a predatorprey system such as (8) is shown in Fig 1B.

The point to address here is that the explicit consideration of two populations growing in mutual dependence originates a system whose range of dynamic behavior is qualitatively different and richer than the single species case. Particularly, the occurrence of oscillatory trajectories of populations can only be explained (in the frame of first-order differential equations models without timelags) through multispecies interaction (see Turchin 2003). On the other hand, complex communities could in principle be decomposed as a net of binary trophic interactions, and therefore the understanding of simple twospecies interactions can provide a basis for understanding real ecological systems. From the above arguments it is clear that twodimensional models are needed to represent a large set of ecological scenarios, and predatorprey systems are particularly important since they form the basis of food webs.

Binary interspecific interactions, and predator-prey models in particular are among the best studied ecological systems from a theoretical standpoint. Besides the mentioned conceptual importance in ecology, twodimensional systems composed by first-order ordinary differential equations receive
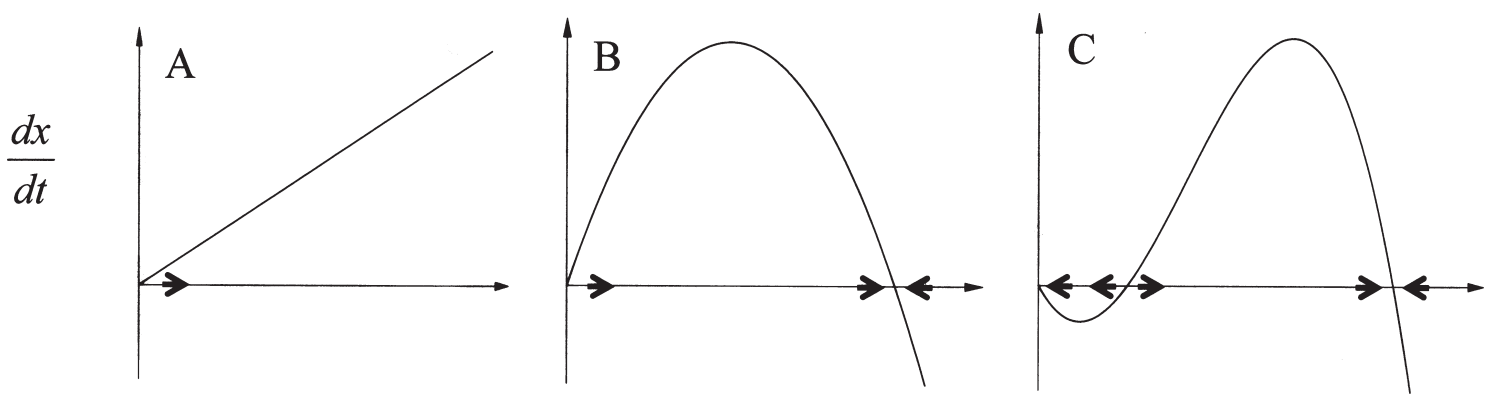

$x$

Fig. 2: One-dimensional phase planes of population models of exponential growth (A), logistic growth (B), and logistic + Allee-effect growth (C).

Planos de fase unidimensionales de modelos poblacionales para crecimiento exponencial (A), crecimiento logístico (B) y crecimiento logístico + efecto Allee (C). 
comparatively large attention due to the fact that mathematical theory is well developed for this kind of dynamical systems. Therefore, there is a good chance to characterize in deepness the dynamic behavior of twodimensional models.

\section{Analysis of two-dimensional model}

In contrast with the one-dimensional case, twodimensional phase-portraits contain both statevariables (Fig. 3). Thus, to locate the equilibria the common procedure is to find the intersections on the plane of the nullclines. This can be accomplished through analytical or numerical methods. We recommend the use of the free-distributing software XPPAUT (Ermentrout 2002) for numerical phase-plane and bifurcation analysis.

Analytically, the stability of the critical points is again obtained by looking at the derivatives of the system's growth rate as a function of the variables at equilibria. Here we are concerned with two-dimensional models whose derivative corresponds to the Jacobian, or community matrix $J(X)$. Ordinary differential equations theory states that the qualitative behavior near the equilibrium point $X^{*}$ is determined by the sign of the real part of the eigenvalues of $J\left(X^{*}\right)$. Since the signs of the real part of eigenvalues depend on the

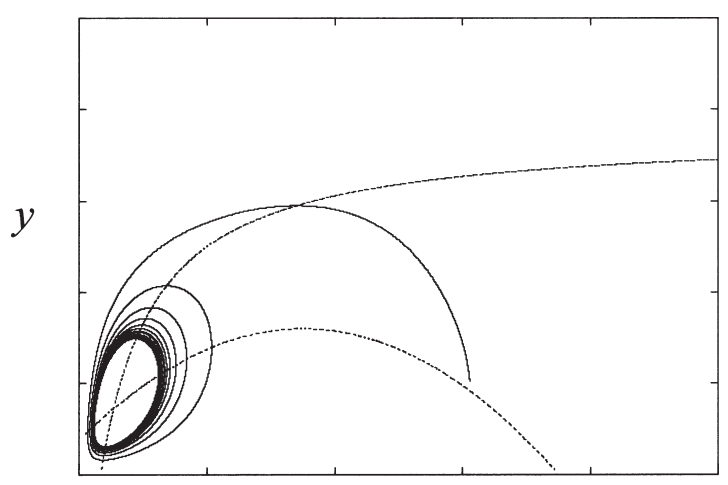

$x$

Fig. 3: Two-dimensional phase-plane of a predator-prey model. The zero-growth isolines are shown, together with a trajectory approaching a limit-cycle.

Plano de fase bidimensional de un modelo depredador-presa. Se muestran las isolíneas de crecimiento cero y una trayectoria que aproxima a un ciclo límite. parameter values contained in the elements of the Jacobian, a change in parameter values could shift those signs from negative to positive or vice versa. The exact parameter values where the sign of the real part of the eigenvalues changes is known as bifurcation set (point, curve or surface), and on this set the real part of one or both eigenvalues is equal to zero. The study of a system at a bifurcation set turns to be highly sophisticated in many cases and can give valuable information about the dynamics of the system (Hainzl 1988). Nevertheless bifurcation points themselves are often considered of lesser importance for ecological purposes since we are mainly interested in the qualitative behavior of the system at different regions of the parameter space, which are delimited by the bifurcation set.

The most important tool available from dynamical system theory for fully analyzing two-dimensional systems is the PoincaréBendixson theorem. It states that given that every solution of the system of differential equations is bounded (satisfied in most ecological models due to population regulation), then any non steady-state trajectory within a closed set presents three possible outcomes: a) the trajectory will approach a steady-state, b) the trajectory is a periodic orbit or c) the trajectory approaches a closed periodic orbit or a cycle graph (see EdelsteinKeshet 1988, Chicone 1999). An important consequence of this theorem is that inside a periodic orbit there must be an equilibrium, which can be stable or unstable. If the orbit surrounding the equilibrium point is a stable limit cycle and the inner point is also stable, there are at least two domain of attraction separated by at least one unstable periodic orbit. Thus, based on the powerful PoincaréBendixson theory, it is possible to fully analyze the dynamical outputs of two-dimensional systems. However, this task can be quite hard when facing two-dimensional biological models with indeed a moderate degree of realism.

Three-dimensional and higher-dimensional models

Often in nature, what is modeled as a binary interaction is a reduced abstraction of a more complex process. For example, exploitative 
competition for food is often represented as a mutually negative interaction between two competitors in circumstances that the system in mind includes a shared resource. Therefore we could better represent an exploitative competition process through extending model (8) to

$$
\begin{aligned}
& \frac{d x}{d t}=r x-b x^{2}-y c x-z h x \\
& \frac{d y}{d t}=p y c x-m y \\
& \frac{d z}{d t}=q z h x-n z
\end{aligned}
$$

(Fig. 1E) where $z$ is the population size of a second predator. As before, more complex conversion and extraction functions will render more sophisticated equations.

Holt (1977) showed that mutually negative effects between two populations could be the result of a process entirely different from competition, where the two apparent competitors are prey which share a common predator (Fig. 1F). These kinds of indirect effects are emergent properties of system of three and higher dimension. Several ecologically important processes can only be represented in models of three or more dimensions, such as trophic cascades (Fig. 1D, see also Kerfoot 1987), and intraguild predation (Polis et al. 1989, Polis \& Holt 1992, Arim \& Marquet 2004, see Fig. 1G). In complex food webs, indirect effects could dominate the system dynamics through reducing, canceling out, or reinforcing the direct effects. Indirect effects can arise either through a chain of direct interactions, or through a modification of a direct binary interaction caused by a third population (Wooton 1994). The final result at the community level depends on the whole feedback structure of the system.

We could generalize somewhat the models already presented in order to include any other functional response (extraction function) and conversion function from food consumed to consumer growth, and to include any possible food web structure.

$$
\frac{d x_{i}}{d t}=x_{i}\left(\sum_{h} g_{i h}\left(\phi_{i h}\right)-\lambda_{i} x_{i}\right)-\sum_{j} x_{j} \phi_{j i}
$$

for any $h \neq i \neq j$, all of them form 1 to $n$. Here $\phi_{i h}$ represents the extraction rate of prey $h$ by one unit of predator $i$. The function $g_{i h}\left(\phi_{i h}\right)$ is the conversion rate of food extracted by the population $i$ from population $h$ to own biomass. Coefficient $\lambda_{i}$ denotes a linear self limitation term.

\section{Analysis of three-dimensional and higher- dimensional models}

Although the ecological relevance of indirect effects and multidimensional systems is well established in the literature, the mathematical analysis of three- and higher-dimensional models is challenging. A fortunate solution of a three-dimensional system is shown in Fig. 4, where a trajectory in a three-dimensional plane approaches a three-dimensional limit cycle.

The main problem when we face a three or higher-dimensional system analysis is that the Poincaré-Bendixson theorem does not applies. This imposes a big difference with the analysis of two-dimensional system, namely, that it is not enough to know the stability of every equilibrium point in order to determine the dynamics of the whole system, since the longterm behavior of a trajectory in a compact set do not necessarily converges to any structure. Furthermore, if non periodic orbits exhibit high-sensitivity to initial conditions, a chaotic

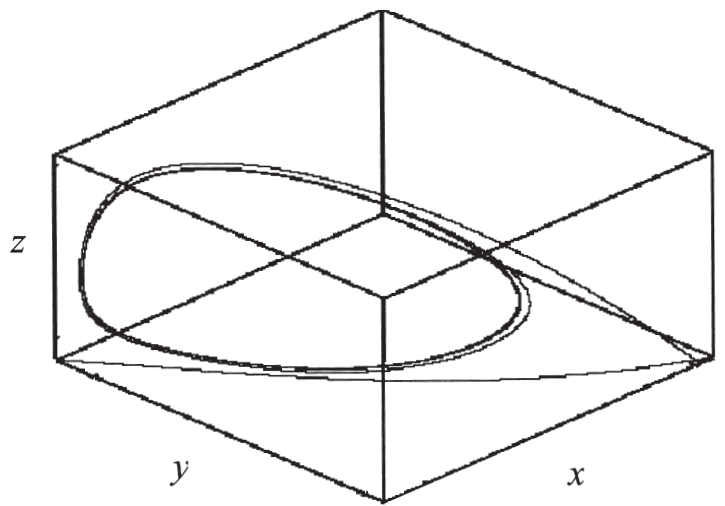

Fig. 4: A solution of a three-dimensional model of the kind shown in Fig. 1F.

Una solución de un modelo tridimensional del tipo mostrado en Fig. 1F. 
curve may appear (see Strogatz 1994). Chaos, together with stable equilibria and stable limit oscillations constitute the best recognized longterm dynamical outputs of population models, and their occurrence in nature is a growing field of contemporary research (Fussmann \& Heber 2002, Jansen \& Kokkoris 2003).

The range of dynamic outputs arising from three-dimensional models, as outlined above, is qualitatively different from that of one- and two-dimensional systems and the ecological meaning of this distinction is relevant. Note that in two-dimensional systems, for instance a predator-prey model, the qualitative asymptotic behavior of one variable is equivalent to that of its partner. This means that if one population approaches a steady-state, the second population also does so; if one population approaches a limit cycle, the second population also oscillates. Conversely, in threedimensional models one of the species can reach a steady-state while the other two are oscillating. An intuitive explanation of this is to consider that a variable at steady-state behaves as a parameter (i.e., the value does not change through time). Therefore, the non-steady-state variables of a $(n)$-dimensional system will behave as those belonging to a (n-k)dimensional system once $k$ of the variables reach a steady-state (for a mathematical proof see Thieme 1992, and see Mena-Lorca \& Hethcote 1992 for an example). As a consequence, a two-dimensional system with one variable closely approaching a steady-state behaves like one-dimensional and cannot exhibit oscillations, but a three-dimensional system with one variable approaching a steadystate will behaves like a two-dimensional one which still allows oscillations (but not chaos). In short, a three-dimensional system can exhibit any combination of stable points with cycles, and cycles with chaos, excepting two variables at steady-state and one cycling. Under the same reasoning, it is easy to elucidate the possible behavior of each variable composing four- or higher-dimensional systems.

The study of local stability properties of a three- and higher-dimensional systems is not an easy task. In order to compute the eigenvalues of an $n$-dimensional Jacobian, we could have to factor out an $n$-degree polynomial, which in the case of $n>4$ there is no explicit formula to find polynomial roots. In this case, it is useful to use the Routh-Hurwitz criteria, which provide conditions for negativity of the polynomial roots. Therefore, this tool sets out the criteria for determining local stability of the equilibrium points without need of calculating the roots of the characteristic polynomial, but instead through manipulating its coefficients. The stability algorithms for verifying stability of two-, three-, four- and five-dimensional models based on the Routh-Hurwitz criteria are summarized in May(1974).

Levins (1974, 1975) developed an approach to apply the Routh-Hurwitz criteria to $n$-dimensional qualitatively-specified systems, known as loop analysis (for recent advances on this theory see Puccia \& Levins 1985, Dambacher et al. 2003a, 2003b). Loop analysis makes use of signed digraphs to compute the conditions for stability and the response of the state-variables to any set of press perturbations exerted on the system's variables. Additionally, loop analysis explains pure mathematical conditions for stability and response prediction in terms of feedback structure of the model system. Given that qualitatively-specified models are easier to build and more amenable to reach agreement with real data, this approach allows studying real multidimensional systems sacrifying precision for the sake of generality and realism (Hulot et al. 2000).

Few fully-specified ecological models of dimension three or higher have been completely studied. The research is often reduced to determine local stability, persistence, invariance and so on; and as mentioned above, from this kind of partial information it is not possible to get a complete picture of the dynamics. In general, the only analytic tool available to study global stability of equilibria is to find a Lyapunov function (Chicone 1999). Briefly, a Lyapunov function is a one that renders level curves surrounding the equilibrium point, and there curves are crossed over by any trajectory toward the equilibrium. Given that such a function exists, one can assure that the trajectories approach to the equilibrium point. In contrast with mechanical and electrical systems where there are natural functions to try (in this case energy), for ecological models there is no standard procedure to build Lyapunov functions. 


\section{Monotone systems}

Recent advances in dynamic system theory show that some special kind of models, the so called monotone dynamical systems, of dimension $n$ will behave as possessing dimension $n-1$ (Smith 1988, 1995, Hirsch \& Smith 2003). Although this theory has important theoretical implications, it is still underappreciated by ecologists.

In ecological terms, competitive and cooperative systems are monotone. A system of populations interacting only via negative effects is said to be competitive, and the reverse holds for a cooperative system. To be more precise, a system which has a Jacobian (community) matrix whose non-diagonal elements are nonpositive is a competitive system. Conversely, a system which has a Jacobian (community) matrix whose non-diagonal elements are nonnegative is cooperative. Therefore, competitive or cooperative two-dimensional systems cannot exhibit sustained oscillations, and competitive or cooperative three-dimensional systems can show cyclic but not chaotic trajectories. On the other hand, this property allow us to use the analytic tools which are functional to systems of the immediately lower dimension. Thus, PoincaréBendixson theory can be employed to analyze competitive or cooperative three-dimensional systems.

Although we just mentioned a sufficient (but not necessary) condition to find competitive and cooperative systems based on the Jacobian matrix, there exist a wider criterion to define monotonicity. A system $X^{\prime}=f(X)$ is said to be competitive (monotone) in the set $D$ if there exists a diagonal matrix $H=\operatorname{diag}\left(\varepsilon_{1}, \varepsilon_{2} \ldots \varepsilon_{n}\right)$ where each $\varepsilon_{i}$ is 1 or -1 , such that the matrix product $H J(X) H$ has only non positive values outside the diagonal, for any $X$ (see Smith 1995). In the above statement, $J(X)$ is the Jacobian matrix of $f$ and $D$ is a convex set (i.e. a set with no holes). Ecological systems that satisfy such conditions are, for example, the Lotka-Volterra two-species competition model.

$$
\begin{aligned}
& \frac{d x}{d t}=r_{x} x\left(1-\frac{x+\alpha_{x y} y}{K_{x}}\right) \\
& \frac{d y}{d t}=r_{y} y\left(1-\frac{x+\alpha_{y x} y}{K_{y}}\right)
\end{aligned}
$$

which is represented in Fig. 1C.
More generally, a system of $n$ competitors of the kind

$$
\frac{d x_{i}}{d t}=r_{i} x_{i}\left(1-\frac{\sum_{j} \alpha_{i j} x_{j}}{K_{i}}\right)=f_{i}\left(x_{1}, \ldots, x_{n}\right)
$$

possesses a Jacobian $J\left(x_{1}, \ldots, x_{n}\right)$ whose nondiagonal elements satisfy the condition

$$
\frac{\partial f_{i}\left(x_{1}, \ldots, x_{n}\right)}{\partial x_{j}}=-\frac{\alpha_{i j} x_{i}}{K_{i}} \leq 0
$$

for every $i \neq . j$, therefore taking $H=\operatorname{diag}(1, \ldots, 1)$ the product $H J(X) H$ has only non-positive values outside the diagonal. Thus, system (12) behaves as a system of dimension $n-1$.

Provided that the Jacobian is sign-stable, any system of $n$ interacting species will be a monotone system if every pair of interacting species have a symmetrical effect between them, irrespective of the existence of one-sided effects (i.e., amensalism or comensalism). More simply, a community of biological populations which exhibits any kind of interaction other than predation or parasitism $(+/-)$ will be a competitive system in the phase space, and will behave as a lower dimensional system. This property can be checked graphically in a loop model if it does not contain any two vertices linked between them by a pair of arcs of opposite sign.

\section{Further considerations about time}

There are two ways how the time can be included into differential equation models, altering in this way the dimension of the system. The first is through incorporating a function explicitly dependent on time. For example, consider a logistic growing population being environmentally forced in a way that one of its parameters (e.g., carrying capacity) is considered to be seasonally changing,

$$
\frac{d x}{d t}=x r\left(1-\frac{x}{k_{o}(1+\cos (a t))}\right)
$$

then the above model is non-autonomous and capable of exhibiting a more complex dynamics than its autonomous counterpart. This model is in fact two-dimensional, even though it has 
only one explicit state-variable. The periodic equation shown above is fully equivalent to the following system of autonomous ODE:

$$
\begin{aligned}
& \frac{d x}{d t}=x r\left(1-\frac{x}{k_{o}(1+\cos (a y))}\right) \\
& \frac{d y}{d t}=1
\end{aligned}
$$

where the two-dimensionality is now entirely apparent.

In general, since any non-autonomous system can be reduced to an autonomous equivalent, the real dimension of the original time-dependent model is that of its associated autonomous. Roughly speaking, by switching an autonomous system into a time-dependent version (e.g., by considering seasonal or inter annual external forcing), one more dimension is being added.

The second way of incorporating the time into differential equation models is through including time-delays, e.g. for modeling the current population growth rate in dependence on the value of the state variable at some time in the past. One particular case of delaydifferential equation is.

$$
\frac{d x(t)}{d t}=x(t) r\left(1-\frac{x(t-\tau)}{K}\right)
$$

This apparently innocent modification to the classical logistic converts the model into infinite-dimensional. For a comparative visualization of this, consider a discrete autoregressive model of the form.

$$
x_{t}=F\left(x_{t-1}, x_{t-2} \ldots x_{t-d}\right)
$$

Here, the state of $x_{t}$ depends on the values of the variables at $d$ previous instants and thus the dimension of this system is $d$ (Berryman 1999, Turchin 2003). In the case of eq. (15) the state of the variable $x(t)$ depends on the values of $x$ at the infinite $(t-s)$ previous instants, for all $s$ in the (continuous) range 0 to $\tau$. Therefore the dimension is infinite whenever time delays are included in a differential equation system, and the expected dynamics is that which belong to higher-dimensional models discussed before. This implies that, for example, a two-variable model can exhibit oscillatory or indeed chaotic behavior in one or both variables, a dynamics only expected in higher-dimensional systems. Conversely, the existence of biological delays within a population can explain the occurrence of complex dynamics without invoking interactions between species. Therefore, simple food web architectures could develop very complex behavior since they are associated to complex feedback structures when time delays are significant.

\section{CONCLUDING REMARKS}

Populations in nature as well as under controlled experiments exhibit a range of endogenous dynamic behavior, from steadystate to periodic and aperiodic oscillations (see empirical examples in Costantino et al. 1997, Berryman 1999, Fussman et al. 2000, Mueller \& Joshi 2000, Turchin 2003). When using mathematical models for explaining ecological processes associated to the dynamics, the dimension of the model system largely influences its dynamical outcome. Although populations in the wild are part of highdimensional ecological networks, it is plausible to assume that through studying a small abstract subset of interacting -or indeed an isolated- species we can gain useful insights about the causes and consequences of their dynamics. Based on a large dataset, Kendall et al. (1998) found that $30 \%$ of the observed time series of animal populations exhibits cyclic behavior, and later on Murdoch et al. (2002) showed that cyclic populations differ in their oscillation period so that many generalist consumers can be adequately described by single-species models. Nevertheless, Yodzis (2000) stressed the inadequacy of lowerdimensional models for represent real networks. At the present state of the ecological theory, it seems that the combined use of one, two, three, and multidimensional models of population dynamics will continue to give us guidelines for basic and applied research.

We stress that the correct consideration of the system dimension is of vital importance when working with theoretical models in ecology. From a theoretical standpoint, given a large initial model, the choice about which variables neglect in order to get a workable model should be based on a former decision 
about the dimension to obtain. Therefore it is necessary to specify the formal dependence of the neglected versus non-neglected variables. In this way the new model is totally defined since the neglected variables are determined by the others. For example, Schaffer (1981) provides a proper method to reduce the number of variables of a model system and obtain what he called an abstracted growth equation. As a simple illustration of this point, consider a system composed by two competing species which share one common predator. If one of the prey is deleted as a variable the original three-dimensional system is reduced to a twodimensional one. Instead, if the predator is the neglected variable the system is reduced to a one behaving as one-dimensional since the resulting community is monotone. On the other hand consider a system of one predator and one prey composed by two stages with fixed ratio (e.g., males and females in constant proportion). This three-variables system is twodimensional because the two stages are not independent variables, and deleting the predator reduces the dimension by one, but deleting one prey stage implies no change in the dimension.

Assuming that statistical tools are available to accurately capture the dominant dimension of a natural system, a minimal model of it should exhibit the observed dimensionality. Once specified the suitable dimension, a set of specific mechanistic hypotheses (alternative models) can be constructed and contrasted according to the concepts discussed here. For example, if the real system is shown to be twodimensional, some model candidates are a second-order single-species model, a simple predator-prey system, and a three-species monotone model, all of them possessing twodimensional behavior. A rigorous analysis of the alternative models can provide further hypotheses to be tested by means of manipulation or subsequent observation in order to reject some alternatives. Once the best model is selected, the mechanism behind the model structure will offer a plausible explanation to the observed natural patterns.

Finally we want to underline that the identification of the real dimension of a dynamical model system in not always obvious, since it is affected by multiple factors others than the number of equations written down.
The dimension is determined by the number of independent state-variables, and it is therefore dependent on the absolute number of variables, their degree of interdependency, their autonomy respect to time, the order of the system, and presence of time-delays. Moreover, structural features such as monotonicity alter the correspondence between dimension and qualitative behavior. On the other hand, depending on the kind of mathematical answer we would want to obtain, the choice of the system's dimension limits our ability to get reliable results. However, analogous drawbacks exist when facing an ecological question by means of empirical tools, since the interactions among sources of variation in experimental settings are arbitrarily bounded by the particular experimental design. As a corollary, we would say that as long as we do not have a solid theory about the evolution of ecological systems (and perhaps we will never have it), serious ecologists should be aware about the limitations of the approaches they use and try to creatively combine the insights arising from diverse lines of attack (including modeling) in order to gain understanding on the questions which motivate their work.

\section{ACKNOWLEDGEMENTS}

The authors thank the support from FONDECYT, grants 1040821 to RRJ and 1990144 to JML. We also acknowledge DIUCV 124789.

\section{LITERATURE CITED}

ARIM M \& PA MARQUET (2004) Intraguild predation: a widespread interaction related to species biology. Ecology Letters 7: 557-564.

BERRYMAN AA (1999) Principles of population dynamics and their application. Stanley Thornes, Cheltenham, United Kingdom. 243 pp.

BERRYMAN AA (2003) On principles, laws and theory in population ecology. Oikos 103: 695-701.

BOUKAL DS \& L BEREC (2002) Single-species models of the Allee effect: extinction boundaries, sex ratio and mate encounters. Journal of Theoretical Biology 218: 375-394.

BRAUER F \& C CASTILLO-CHÁVEZ (2001) Mathematical models in population biology and epidemiology. Springer-Verlag, New York, New York, USA. 416 pp.

CHICONE C (1999) Ordinary differential equations with applications. Springer-Verlag, New York, New York, USA. 561 pp. 
COSTANTINO RF, DESHARNAIS RA CUSHING JM \& B DENNIS (1997) Chaotic dynamics in an insect population. Science 275: 389-391.

DAMBACHER JM, LI HW \& PA ROSSIGNOL (2003a) Qualitative predictions in model ecosystems. Ecological Modelling 161: 79-93.

DAMBACHER JM, LUH H, LI HW \& PA ROSSIGNOL (2003b) Qualitative stability and ambiguity in model ecosystems. American Naturalist 161: 876-888.

DENNIS B (1989) Allee effects: population growth, critical density, and the chance of extinction. Natural Resource Modeling 3: 481-538.

EDELSTEIN-KESHET L (1988) Mathematical models in biology. McGraw-Hill, New York, New York, USA. 586 pp.

ERMENTROUT B (2002) Simulating, analyzing, and animating dynamical systems. A guide to XPPAUT for researchers and students. SIAM, Philadelphia, USA. 290 pp.

FUSSMANN GF, ELLNER SP, SHERTZER KW \& HAIRSTON N (2000) Crossing the Hopf bifurcation in a live predator-prey system. Science 290: $1358-1360$.

FUSSMANN GF \& G HEBER (2002) Food web complexity and chaotic population dynamics. Ecology Letters 5: 394-401.

GAO LQ, MENA-LORCA J \& HW HETHCOTE (1996) Variations on a theme of SEI endemic models. In: Martelli M, Cooke K, Cumberbatch E, Tang B \& H Thieme (eds) Differential equations and applications to biology and industry: 191-207. World Scientific, Singapore, Malaysia.

GETZ WM (1991) A unified approach to multispecies modeling. Natural Resource Modeling 5: 393-421.

GETZ WM (1993) Metaphysiological and evolutionary dynamics of populations exploiting constant and interactive resources: $\mathrm{r}-\mathrm{K}$ selection revisited. Evolutionary Ecology 7: 287-305.

GETZ WM (2003) Is population ecology a mature science? Bioscience 53: 885-888.

GINZBURG LR (1998) Assuming reproduction to be a function of consumption raises doubts about some popular predator-prey models. Journal of Animal Ecology 67: 325-327.

GINZBURG LR \& M COLYVAN (2004) Ecological orbits: how planets move and populations grow. Oxford University Press, New York, New York, USA. 166 pp.

GURNEY WSC \& RM NISBET (1998) Ecological dynamics. Oxford University Press, New York, New York, USA. 335 pp.

HAINZL (1988) Stability and Hopf bifurcation in a predator-prey system with several parameters. SIAM Journal on Applied Mathematics 48: 170190.

HETHCOTE HW (2000) The Mathematics of Infectious Diseases. SIAM Review 42: 599-653.

HIRSCH MW \& HL SMITH (2003) Monotone systems, a mini-review, in positive systems. In: Benvenuti L, De Santis A \& L Farina (eds) Lecture notes on control and information sciences: 183-190. Springer-Verlag, Heidelberg, Germany.

HOLT RD (1977) Predation, apparent competition, and the structure of prey communities. Theoretical Population Biology 12: 197-229.

HULOT FD, LACROIX G, LESCHER-MOUTOUÉ \& M LOREAU (2000) Functional diversity governs ecosystem response to nutrient enrichment. Nature 405: 340-344.

JANSEN VAA \& GD KOKKORIS (2003) Complexity and stability revisited. Ecology Letters 6: 498-502.
KENDALL BE, PRENDERGAST J \& ON BJORNSTAD (1998) The macroecology of population dynamics: taxonomic and biogeographical patterns in population cycles. Ecology Letters 1: 160-164.

KERFOOT WC (1987) Cascading effects and indirect pathways. In: Kerfoot WC \& A Sih (eds) Predation, direct and indirect impacts on aquatic communities: 57-70. University Press of New England, Hanover, New Hampshire, USA.

KOT M (2001) Elements of mathematical ecology. Cambridge University Press, Cambridge, United Kingdom. 464 pp.

MUELLER LD \& A JOSHI (2000) Stability in model populations. Princeton University Press, Princeton, New Jersey, USA. 319 pp.

LEVINS R (1968) Evolution in changing environments. Some theoretical explorations. Princeton University Press, Princeton, New Jersey, USA. 120 pp.

LEVINS R (1974) The qualitative analysis of partially specified systems. Annals of the New York Academy of Sciences 231: 123-138.

LEVINS R (1975) Evolution of communities near equilibrium. In: Cody M \& JM Diamond (eds) Ecology and evolution of communities: 16-50. Harvard University Press, Cambridge, Massachusetts, USA.

MARQUET PA \& JX VELASCO-HERNÁNDEZ (1997) A source-sink patch occupancy metapopulation model. Revista Chilena de Historia Natural 70: 371 380.

MAY RM (1974) Stability and complexity in model ecosystems. Second edition. Princeton University Press, Princeton, New Jersey, USA. 265 pp.

MENA-LORCA J \& HW HETHCOTE (1992) Dynamic models of infectious disease as regulators of populations sizes. Journal of Mathematical Biology 30: 693-716.

MENA-LORCA J, VELASCO-HERNÁNDEZ JX \& C CASTILLO-CHAVEZ (1999) Density-dependent dynamics and superinfection in epidemic models. IMA Journal of Mathematics Applied to Medicine and Biology 16: 307-317.

MURDOCH WW, KENDALL BE, NISBET RM, BRIGGS CJ, McCAULEY E \& R BOLSER (2002) Singlespecies models for many-species food webs. Nature 417: 541-543.

POLIS GA \& RD HOLT (1992) Intragulid predation: the dynamics of complex trophic interactions. Trends in Ecology and Evolution 7: 151-155.

POLIS GA, MYERS CA \& RD HOLT (1989) The ecology and evolution of intraguild predation: potential competitors that eat each other. Annual Review of Ecology and Systematics 20: 297-330.

PUCCIA CJ \& R LEVINS (1985) Qualitative modeling of complex systems. An introduction to loop analysis and time averaging. Harvard University Press, Cambridge, Massachusetts, USA. 259 pp.

RAMOS-JILIBERTO R (2005) Resource-consumer models and the biomass conversion principle. Environmental Modelling \& Software 20: 85-91.

ROYAMA T (1977) Population persistence and density dependence. Ecological Monographs 47: 1-35.

ROYAMA T (1992) Analytical population dynamics. Chapman and Hall, London, United Kingdom. 371 pp.

SCHAFFER WM (1981) Ecological abstraction: the consequences of reduced dimensionality in ecological models. Ecology 51: 383-401.

SMITH HL (1988) Systems of ordinary differential equations which generate an order preserving flow, a survey of results. SIAM Review 30: 87-113. 
SMITH HL (1995) Monotone dynamical systems. An introduction to the theory of competitive and cooperative systems. Mathematical Surveys and Monographs, volume 41, American Mathematical Society, Providence, Rhode Island, USA. 174 pp.

STEPHENS PA \& WJ SUTHERLAND (1999) Consequences of the Allee effect for behavior, ecology and conservation. Trends in Ecology and Evolution 14: 401-405.

STROGATZ SH (1994) Nonlinear dynamics and chaos. Perseus Publishing, Cambridge, Massachusetts, USA. $498 \mathrm{pp}$

THIEME HR (1992) Convergence results and a Poincaré-
Bendixson trichotomy for asymptotically autonomous differential equations, Journal of Mathematical Biology 30: 755-763.

TURCHIN P (2003) Complex population dynamics: a theoretical/empirical synthesis. Princeton University Press, Princeton, New Jersey, USA. 450 pp.

WOOTTON T (1994). The nature and consequences of indirect effects in ecological communities. Annual Review of Ecology and Systematics 25: 443-66.

YODZIS P (1989) Introduction to theoretical ecology. Harper \& Row, New York, USA. 384 pp.

YODZIS P (2000) Diffuse effects in food webs. Ecology 81: 261-266. 